\title{
Association of rare PTP4A1-PHF3-EYS variants with alcohol dependence
}

\author{
Journal of Human Genetics (2013) 58, 178-179; doi:10.1038/jhg.2012.153; published online 17 January 2013
}

In a recent genome-wide association study (GWAS), we identified a unique novel, functional and replicable risk genomic region for alcohol dependence. This region (Chr6: 64,066,604-64,831,120) included the protein tyrosine phosphatase type IVA 1 gene (PTP4A1), the plant homeodomain finger protein 3 gene $(P H F 3)$ and part of the eyes shut homolog gene (EYS). It was enriched with numerous replicable common risk variants (minor allele frequency $(\mathrm{MAF})>0.05$ ) for alcohol dependence across AfricanAmericans, European-Americans and European-Australians. Within $90 \mathrm{Mb}$ range surrounding this region in the discovery sample, all variants with $P<10^{-4}$ were concentrated in this region. Most of these risk variants had significant cis-acting regulatory effects on mRNA expression. The distributions of $-\log (P)$ values for association and functional signals in this region were highly consistent across six independent populations. We thus speculated that this region might harbor a causal variant(s) for alcohol dependence. $^{1}$

Rare variants, which may not be wellcaptured by a GWAS, may be of equal importance as common variants in the etiology of complex disorders. Recent studies have indicated that rare variants, which usually have strong effects and high penetrance, are involved in the genetic susceptibility to an increasing number of human diseases or traits; for example, plasma levels of high-density lipoprotein cholesterol $^{2}$ and type 1 diabetes $^{3}$ as well complex neuropsychiatric disorders such as autism. ${ }^{4-6}$ However, the associations between rare variants within the PTP4A1-PHF3-EYS region and alcohol dependence have never been tested. Furthermore, alcohol dependence has high rates of comorbidity with numerous neuropsychiatric disorders, including anxiety disorders, major depression, bipolar disorders, schizophrenia, post-traumatic stress disorder and so on. The associations between rare PTP4A1-PHF3-EYS variants and these neuropsychiatric disorders have never been tested either.

To further explore the role of PTP4A1PHF3-EYS region in alcohol dependence, in the present study, we imputed the untyped variants in this PTP4A1-PHF3-EYS region across 21 independent cohorts with 11 different neuropsychiatric disorders (Table 1), and then examined the associations between rare PTP4A1-PHF3-EYS variants $(0<\mathrm{MAF}$ $<0.05$ in controls) and these disorders, so that we could test whether rare PTP4A1PHF3-EYS variants were associated with alcohol dependence and whether these associations were specific for alcohol dependence. The data of these disorders were all of those with neuropsychiatric and neurological disorders available for our analysis from the dbGaP database (http://www.ncbi.nlm.nih. gov/gap/).

A total of 49268 subjects in these 21 cohorts were analyzed (Table 1), including 10554 subjects in 3 cohorts with alcohol dependence (DSM-IV) and 38714 subjects in other 18 non-alcoholism cohorts. The European-Australian cohort with alcohol dependence included 871 parents, 1645 affected offspring and 3922 unaffected offspring. Detailed demographic information for these samples has been published (Supplementary Table S1). ${ }^{1,7-9}$ We used the same strategies as previously to maximize the success rate and accuracy of imputation and to stringently clean the phenotype and genotype data. ${ }^{8,9}$ Associations between individual variants and diseases were tested using the program PLINK (for case-control data) and FBAT (for family-based data)., ${ }^{8,9}$ The experiment-wide significance levels $(\alpha)$ were set at $1.4 \times 10^{-5}$ (in AAs) and
$2.1 \times 10^{-5}$ (in EAs) for these individual rare variant analyses based on the correction for the numbers of effective genetic markers $(n=175,115$ in AAs and EAs, respectively; calculated from the entire marker set by the adjusted Bonferroni-type program SNPSpD) and the number of cohorts (that is, $n=21$ ). Furthermore, associations between rare variant constellations across the entire PTP4A1PHF3-EYS region and diseases were tested using the Variable MAF Threshold (VT) test implemented in the program SCORE-Seq, ${ }^{9}$ in order to explore the synthetic effects of rare variants within the entire region. In VT test, the weight was set at 1 in the regression model, while the MAF threshold varied between 0 and 0.05 . Finally, a total of 197956 single-nucleotide polymorphisms (SNPs) with $0<\mathrm{MAF}<0.05$ (in controls) were extracted for association analysis. The MAFs and minimal $P$-values of the most significant risk SNPs, the SNP numbers with $P<0.05$ and the $P$-values for VT tests are shown in Table 1.

We found a rare variant constellation across the entire region that was significantly associated with alcohol dependence in European-Australians $\left(P=4.2 \times 10^{-3}\right)$, and modestly associated with schizophrenia in European-Americans (in GAIN data: $P=0.033$ ), but not with any other neuropsychiatric disease examined $(P>0.10)$. Additionally, single-point association analysis showed that $0-108$ individual SNPs were nominally associated with these 11 diseases $(P<0.05)$, but none of them were significant after experiment-wide correction (Table 1).

We confirmed that rare variants within the PTP4A1-PHF3-EYS region had significant synthetic effects on alcohol dependence in European-Australians, consistent with our previous findings in a GWAS. Both studies support our conclusion that this region 
Table 1 Associations between rare PTP4A1-PHF3-EYS variants and different neuropsychiatric disorders

\begin{tabular}{|c|c|c|c|c|c|c|c|c|c|c|c|c|c|}
\hline \multirow[b]{2}{*}{ Human diseases } & \multirow[b]{2}{*}{ Ethnicity } & \multirow[b]{2}{*}{ Design } & \multirow[b]{2}{*}{ Data set name } & \multirow{2}{*}{$\begin{array}{l}\text { SNP \# } \\
\text { (total) }\end{array}$} & \multirow{2}{*}{$\begin{array}{c}\text { SNP \# } \\
(\mathrm{P}<0.05)\end{array}$} & \multirow{2}{*}{$\begin{array}{l}\text { VT test } \\
\text { P-value }\end{array}$} & \multirow{2}{*}{$\begin{array}{l}\text { Minimal } \\
\text { P-value }\end{array}$} & \multirow{2}{*}{$\begin{array}{c}\text { Most sig. } \\
\text { SNP }\end{array}$} & \multirow[b]{2}{*}{ Gene } & \multicolumn{2}{|c|}{ Affected } & \multicolumn{2}{|c|}{ Unaffected } \\
\hline & & & & & & & & & & $\mathrm{N}$ & MAF & $\mathrm{N}$ & MAF \\
\hline Alcoholism & $\mathrm{AA}$ & $\mathrm{CC}$ & SAGE + COGA & 956 & 22 & 0.335 & $9.5 \times 10^{-4}$ & rs319919 & EYS & 681 & 0.071 & 508 & 0.039 \\
\hline Alcoholism & EA & $\mathrm{CC}$ & SAGE + COGA & 467 & 17 & 0.230 & 0.015 & rs114282789 & EYS & 1409 & 0.016 & 1518 & 0.008 \\
\hline Schizophrenia & EA & $\mathrm{CC}$ & GAIN & 251 & 26 & 0.033 & 0.018 & rs72872860 & $E Y S$ & 1351 & 0.011 & 1378 & 0.019 \\
\hline Bipolar disorder & EA & $\mathrm{CC}$ & $\mathrm{BDO}+\mathrm{GRU}$ & 197 & 4 & 0.685 & 0.015 & rs1779781 & 5' to PTP4A1 & 368 & 0.051 & 1034 & 0.029 \\
\hline Bipolar disorder & EA & $\mathrm{CC}$ & BARD + GRU & 213 & 1 & 0.836 & 0.019 & rs6914590 & PHF3 & 653 & 0.009 & 1034 & 0.002 \\
\hline Bipolar disorder & AA & $\mathrm{CC}$ & BARD + GRU & 618 & 2 & 0.350 & 0.032 & rs1779776 & 5' to PTP4A1 & 141 & 0.021 & 671 & 0.007 \\
\hline ADHD & $\mathrm{CA}$ & Fam & IMAGE & 439 & 73 & 0.566 & 0.001 & rs115865360 & EYS & 924 & 0.026 & 924 & 0.033 \\
\hline Autism & EA & Fam & AGP & 488 & 36 & 0.261 & $1.6 \times 10^{-4}$ & rs116469676 & $E Y S$ & 1330 & 0.004 & 1330 & 0.068 \\
\hline Major depression & $\mathrm{CA}$ & $\mathrm{CC}$ & PRSC & 470 & 108 & 0.516 & $1.4 \times 10^{-4}$ & rs76370578 & 5' to PTP $4 A 1$ & 1805 & 0.040 & 1820 & 0.021 \\
\hline Early onset stroke & AA & $\mathrm{CC}$ & GEOS $\times 3$ & 936 & 43 & 0.789 & 0.003 & rs115889991 & $E Y S$ & 309 & 0.046 & 290 & 0.009 \\
\hline Ischemic stroke & $\mathrm{CA}$ & $\mathrm{CC}$ & ISGS & 367 & 6 & 0.830 & 0.009 & rs116215749 & 3' to PTP $4 A 1$ & 219 & 0.044 & 266 & 0.013 \\
\hline Parkinson's disease & CA & $\mathrm{CC}$ & NGRC & 516 & 9 & 0.819 & $4.0 \times 10^{-4}$ & rs80059369 & EYS & 2000 & 0.031 & 1986 & 0.048 \\
\hline Parkinson's disease & $\mathrm{CA}$ & $\mathrm{CC}$ & PDRD + GRU & 459 & 1 & 0.928 & 0.048 & rs79831694 & EYS & 900 & 0.017 & 867 & 0.033 \\
\hline Parkinson's disease & CA & CC & Ing_coriell_pd & 462 & 11 & 0.749 & 0.007 & rs72875016 & EYS & 940 & 0.059 & 801 & 0.035 \\
\hline
\end{tabular}

Abbreviations: AA, African-American; EA, European-American; EAu, European-Australian; CA, Caucasian; CC, case-control design; MAF, minor allele frequency; N, sample size; SNP, singlenucleotide polymorphism; VT, Variable MAF Threshold test (using SCORE-Seq).

Only the most significant risk markers are listed. The corrected significance levels ( $\alpha$ ) are set at $1.4 \times 10^{-5}$ (in AAs) and $2.1 \times 10^{-5}$ (in EAs) based on correction for the numbers of effective genetic markers $(n=175,115$ in AAs and EAs, respectively; calculated by SNPSpD) and the number of cohorts (that is, 21$)$. $P$ values $<0.05$ in VT test are bold.

Data set names correspond to dbGaP. In family-based cohorts, $N=$ sample size of offspring; 'affected MAF' = 'transmitted MAF', 'unaffected MAF' = 'untransmitted MAF' in offspring.

might harbor a causal variant(s) for alcohol dependence. Additionally, the rare variant constellation was also modestly associated with schizophrenia in European-Americans, but not with any other neuropsychiatric disorder examined. This genetic commonality between alcohol dependence and schizophrenia may underlie high rate of comorbidity between them. It has been reported that over one-third of patients with schizophrenia have an alcohol use disorder. ${ }^{10}$ Our findings support us to postulate that the underlying neuropathological abnormalities of schizophrenia related to the PTP4A1PHF3-EYS variants might potentially facilitate the positive reinforcing effects of alcohol use. Finally, we admit that, as a future work, the imputed genotype data in this study need to be validated by a real molecular experiment, more neuropsychiatric disorders comorbid with alcohol dependence need to be examined, and the current findings warrant replication in independent cohorts.
Lingjun Zuo ${ }^{1}$, Xiangyang Zhang ${ }^{2}$, Hong-wen Deng ${ }^{3}$ and Xingguang Luo ${ }^{1}$

${ }^{1}$ Department of Psychiatry, Yale University School of Medicine, New Haven, CT, USA; ${ }^{2}$ Menninger Department of Psychiatry and

Behavioral Sciences, Baylor College of Medicine, Houston, TX, USA and ${ }^{3}$ Department of Biostatistics, School of Public Health and Tropical Medicine, Tulane University, New Orleans, LA, USA E-mail: Lingjun.Zuo@yale.edu orXingguang.Luo@yale.edu

1 Zuo, L., Zhang, C. K., Wang, F., Li, C. S., Zhao, H., Lu, L. et al. A novel, functional and replicable risk gene region for alcohol dependence identified by genome-wide association study. PLoS One 6, e26726 (2011).

2 Cohen, J. C., Kiss, R. S., Pertsemlidis, A., Marcel, Y. L., McPherson, R. \& Hobbs, H. H. Multiple rare alleles contribute to low plasma levels of HDL cholesterol. Science 305, 869-872 (2004).

3 Nejentsev, S., Walker, N., Riches, D., Egholm, M. \& Todd, J. A. Rare variants of IFIH1, a gene implicated in antiviral responses, protect against type 1 diabetes. Science 324, 387-389 (2009).

4 Sanders, S. J., Murtha, M. T., Gupta, A. R., Murdoch, J. D., Raubeson, M. J., Willsey, A. J. et al. De novo mutations revealed by whole-exome sequencing are strongly associated with autism. Nature 485, 237241 (2012).

5 Neale, B. M., Kou, Y., Liu, L., Ma'ayan, A., Samocha, K. E., Sabo, A. et al. Patterns and rates of exonic de novo mutations in autism spectrum disorders. Nature 485, 242-245 (2012).

6 O'Roak, B. J., Vives, L., Girirajan, S., Karakoc, E., Krumm, N., Coe, B. P. et al. Sporadic autism exomes reveal a highly interconnected protein network of de novo mutations. Nature 485, 246-250 (2012).

7 Zuo, L., Gelernter, J., Zhang, C. K., Zhao, H., Lu, L., Kranzler, H. R. et al. Genome-wide association study of alcohol dependence implicates KIAA0040 on chromosome 1q. Neuropsychopharmacology 37, 557-566 (2011).

8 Zuo, L., Zhang, X. Y., Wang, F., Li, C. S. R., Lu, L., Ye, L. et al. Genome-wide significant association signals in IP011-HTR1A region specific for alcohol and nicotine codependence. Alcohol. Clin. Exp. Res. (e-pub ahead of print 6 December 2012; doi:10.1111/acer.12032).

9 Zuo, L., Zhang, H., Malison, R. T., Li, C. S., Zhang, X. Y., Wang, F. et al. Rare ADH variant constellations are specific for alcohol dependence. Alcohol Alcohol. (e-pub ahead of print 27 September 2012; doi:10.1093/alcalc/ags104).

10 Green, A. I. \& Brown, E. S. Comorbid schizophrenia and substance abuse. J. Clin. Psychiatry 67, e08 (2006).

Supplementary Information accompanies the paper on Journal of Human Genetics website (http://www.nature.com/jhg) 